\title{
Two-year viral load suppression among adolescents receiving antiretroviral therapy in the Cape Metropole, South Africa, 2013 - 2015: A retrospective cohort analysis
}

\author{
B E van Wyk, ${ }^{1}$ MSc, DPhil; E Kriel, ${ }^{1,2} \mathrm{MB}$ ChB, MPH; F C Mukumbang, ${ }^{1}$ MNUR, PhD \\ ${ }^{1}$ School of Public Health, Faculty of Community and Health Sciences, University of the Western Cape, Cape Town, South Africa \\ ${ }^{2}$ Department of Health, Western Cape Government, Cape Town, South Africa
}

Corresponding author: B Evan Wyk(bvanwyk@uwc.ac.za)

\begin{abstract}
Background. In 2018, 4\% of all people living with HIV globally were adolescents aged $10-19$ years. It is reported that adolescents on antiretroviral therapy (ART) are at increased risk of poor viral load suppression (VLS) compared with children and adults.

Objectives. To determine the 24-month prevalence of VLS among adolescents initiated on ART in 2013 in public health facilities in the Metro District Health Services of Western Cape Province, South Africa.

Methods. A retrospective cohort analysis was done on 220 adolescents initiated on ART in 2013; they were followed up for 24 months. Data were extracted from the provincial Tier.Net electronic database and patients' clinical records, and captured in Excel. SPSS statistical software was used for descriptive and inferential analysis; bivariate analysis was used to determine significance and strength of associations between VLS and various sociodemographic and clinical characteristics at 4, 12 and 24 months (with significance set at $p<0.05$ ).

Results. VLS declined over the study period, with rates of 59.5\%, $40.0 \%$ and $25.0 \%$ at 4,12 and 24 months post initiation on treatment, respectively. In bivariate analysis, VLS was significantly associated with younger age (10 - 14 years) at month 12 (crude risk ratio (RR) 1.83 (95\% confidence interval (CI) 1.35 - 2.49)) and month 24 (RR 3.38 (95\% CI 2.24 - 5.10)) after initiation on ART. Male adolescents were significantly more likely to achieve VLS than females (month 4: RR 1.49 (95\% CI 1.22 - 1.81); month 12: RR 1.50 (95\% CI 1.07 - 2.12); and month 24: RR 2.33 (95\% CI 1.50 - 3.62)). Pregnant adolescents were significantly less likely to attain VLS (month 4: RR 0.69 (95\% CI 0.53 - 0.89); month 12: RR 0.64 (95\% CI 0.44 - 0.94); and month 24: RR 0.24 (95\% CI 0.11 - 0.50)) compared with those who were not pregnant. However, of those adolescents who were retained in care, VLS was higher at months 4, 12 and 24 , with rates of $86.8 \%, 79.3 \%$ and $68.8 \%$, respectively. Conclusions. Targeted interventions are required to improve retention in care and VLS among adolescents on ART, with specific focus on older (15 - 19-year-olds) and pregnant adolescents.
\end{abstract}

S Afr Med J 2020;110(12):1213-1217. https://doi.org/10.7196/SAMJ.2020.v110i12.14509

In 2018, it was estimated that 1.6 million adolescents between the ages of 10 and 19 years, globally, were infected with HIV, accounting for $4 \%$ of all people living with HIV. ${ }^{[1]}$ The United Nations Children's Fund (UNICEF) reported that of the estimated 770000 people who died of AIDS-related illnesses, 33000 were adolescents, comprising $4 \%$ of total deaths in 2018. Sub-Saharan Africa (SSA) has the highest number of HIV-infected adolescents ( 1.5 million $(89 \%))$. It is estimated that 310000 adolescents were living with HIV in South Africa (SA) in 2018, with 24 per 100000 annual AIDS-related deaths.

An alarming trend over the past decade is the increase in AIDSrelated deaths among adolescents, which contrasts with the decrease in deaths among all other age groups. ${ }^{[2]}$ Globally, AIDS-related deaths decreased by almost $40 \%$ between 2005 and 2013 for all age groups, except among adolescents (aged 10 - 19 years). HIV is the leading cause of adolescent mortality in SSA and the second highest worldwide. ${ }^{[3]}$ In 2012, the leading cause of death in all age groups in SA was HIV/ AIDS: 50.7\% among children (5 - 14 years) and 51.9\% among adults (15 - 44 years). ${ }^{[4]}$ The national household HIV study estimated that 131052 children (0 - 14 years) and 273981 adolescents and young adults (15 - 24 years) had been exposed to antiretroviral therapy (ART) in 2017 , and $51.9 \%$ and $47.7 \%$ had achieved viral load suppression (VLS), respectively. ${ }^{[5]}$

Although SSA has recorded comparably better adherence rates among adolescents on ART than other regions, ${ }^{[6]}$ the main treatment outcome - VLS - remains unsatisfactory. ${ }^{[-9]}$ A meta-analysis of 8 studies in SA reported VLS among adolescents and young adults receiving ART as $81 \% \cdot{ }^{[10]}$ Earlier studies conducted in SA reported VLS among adolescents at 12 months as $76 \%$ in selected public clinics in Gauteng and Mpumalanga provinces between 2004 and 2010; and 27\% in a Cape Town-based ART clinic between 2002 and 2009.

Despite the poor treatment outcomes regarding VLS displayed by adolescents receiving ART, their situations are often overlooked because routine reporting in HIV programmes focus mainly on outcomes for paediatric $(0-14$ years) and adult ( $\geq 15$ years) populations. Understanding treatment outcomes for adolescents (10 19 years) is critical, because during this period patients are made aware of their HIV status (infected vertically), and have to learn to self-manage their disease (i.e. adhere to treatment regimens and attend clinic appointments) as they are transitioned to the adult HIV treatment programme.

\section{Objectives}

We report findings on 2-year treatment outcomes (VLS) of adolescent patients who were newly initiated on ART in public health facilities in the Metropole of Western Cape Province in 2013, and the risk factors associated with VLS at 4,12 and 24 months after ART initiation.

\section{Methods}

Design

We conducted a retrospective cohort analysis of adolescents aged 
10 - 19 years, who were initiated on ART in 2013 in public health facilities in the Western Cape Metropole.

\section{Participants}

Data from 332 adolescent patients on ART from 29 facilities spread across the Western Cape Metropole were obtained from the provincial Tier.Net register. After cleaning the data, 220 adolescents were included in the final analysis. Of the 112 that were excluded, 68 medical records could not be found, despite numerous attempts at tracing these documents at the various facilities. Furthermore, 28 patients were incorrectly captured as new patients (when they were transferred from other facilities); 4 patients were not adolescents (dates of birth incorrectly captured); and 12 patients had been incorrectly captured as having initiated ART in 2013. Fig. 1 illustrates the sampling process.

\section{Main outcome measures and} analysis

We extracted data on sociodemographic characteristics (age, sex, source of income, type of dwelling, disclosure to significant other and reported alcohol or other drug use) and clinical characteristics (CD4 count, World Health Organization (WHO) clinical stage, tuberculosis (TB) treatment, being pregnant, isoniazid preventive therapy (IPT), co-trimoxazole use and ART regimen).

Bivariate analysis was conducted to determine the significance and strength of associations between VLS at 4, 12 and 24 months and various sociodemographic (age, sex and being pregnant) and clinical characteristics (WHO staging). Statistical significance was established using the $\chi^{2}$ test (with significance set at $p<0.05$ ) and where significance was observed, strength of associations was calculated as risk ratios with 95\% confidence intervals (CIs), using SPSS version 26 (IBM Corp., USA).

\section{Ethical approval}

The protocol was approved by the University of the Western Cape Biomedical Research Ethics Committee (ref. no. BM/17/1/15) and the Western Cape Government Health Impact Assessment Committee (ref. no. WC_2017RP58_418). Permission to access patients' clinical records from the various healthcare facilities was obtained from the facility managers. No informed consent was required, as there was no direct contact with patients and no patient identifiers were collected.

\section{Results}

Of the 220 patients who were newly initiated on ART in 2013, the majority were older adolescents ( $15-19$ years) $(n=179 ; 81.4 \%)$ and female $(n=182 ; 82.7 \%)$ (Table 1$)$. Most adolescents included in the study received financial support from their families and friends $(n=129 ; 58.6 \%)$ and lived in a formal house $(n=116 ; 52.7 \%)$. In line with the HIV clinical treatment guidelines, the overwhelming majority $(n=182 ; 87 \%)$ had disclosed their HIV status to a significant other, or in the case of younger adolescents, HIV status had been disclosed to them.

The median CD4 count at ART initiation was 292.5 cells $/ \mu \mathrm{L}$ (interquartile range (IQR) 228.8 - 391.3). No baseline CD4 count had been recorded for 2 participants. Half of the participants $(n=109)$ had CD4 counts of 200 -

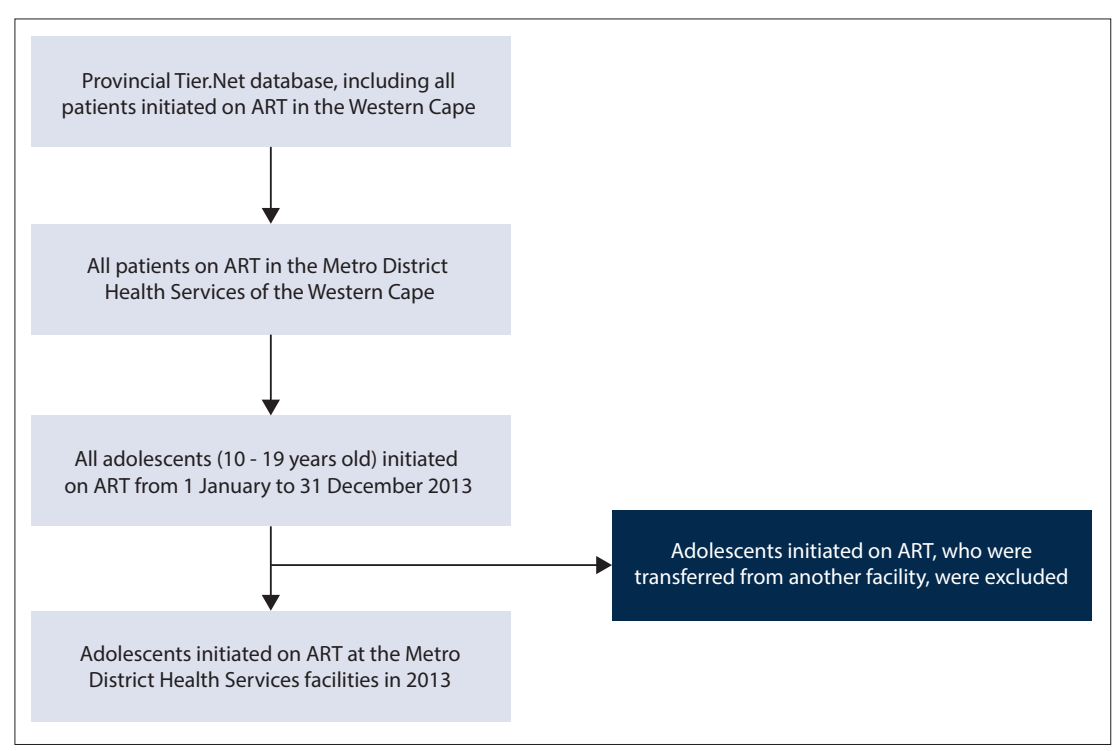

Fig. 1. Sampling process. $(A R T=$ antiretroviral therapy. $)$

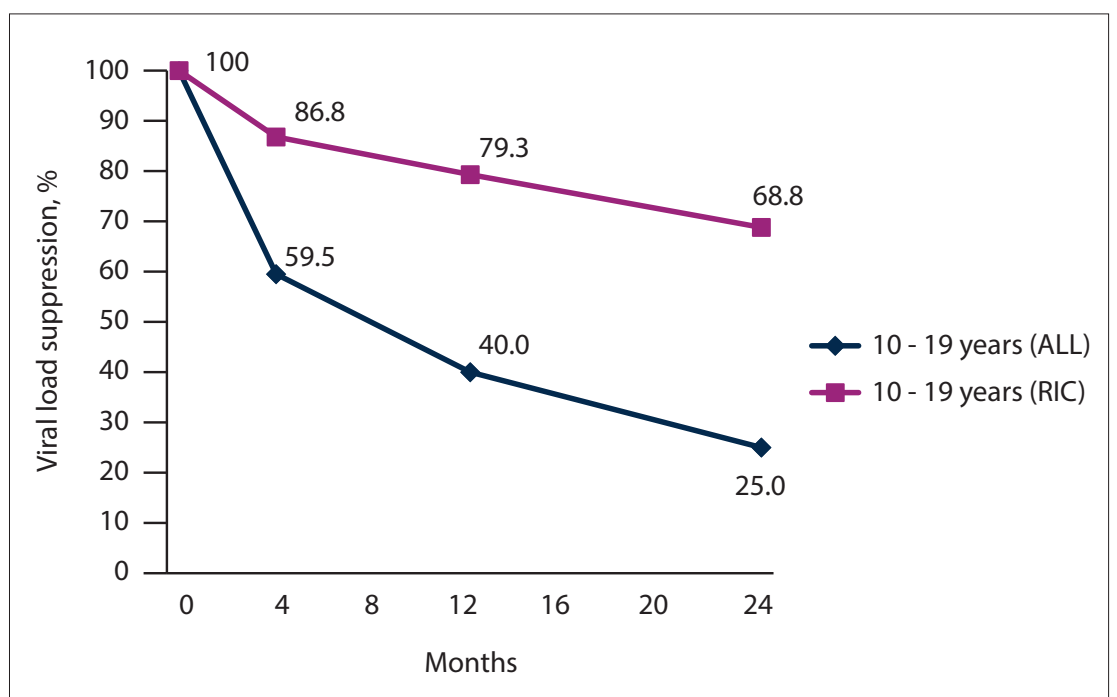

Fig. 2. Comparison of viral load suppression in all adolescents $v$. those retained in care. $(A L L=$ all; RIC $=$ retained in care. $)$

349 cells $/ \mu \mathrm{L}$, and $19 \%(n=42)$ had baseline CD4 counts $<200$ cells $/ \mu \mathrm{L}$.

Of the 213 participants who had WHO staging done at ART initiation, $46.5 \%$ and $22.5 \%$ were WHO stage I and II, respectively; and $23.2 \%$ and $6.8 \%$ were WHO stage III and IV, respectively.

Loss to follow-up was considerable, with only $151(68.6 \%), 111(50.5 \%)$ and 80 $(36.4 \%)$ retained in care at 4,12 and 24 months after initiation, respectively.

VLS was low throughout the study period, with $131(59.5 \%), 88(40 \%)$ and $55(25 \%)$ adolescents having recorded VLS at 4,12 and 24 months after ART initiation, respectively. Fig. 2 illustrates the comparison of VLS at 4, 12 and 24 months post-initiation VLS for all adolescents compared with those who remained in care at the various time points 
Table 1. Characteristics of adolescents initiated on ART with viral load suppression at Metro District Health Services facilities in the Western Cape, South Africa, 2013

\begin{tabular}{|c|c|c|c|c|c|c|c|}
\hline \multirow[b]{2}{*}{ Characteristics } & \multirow[b]{2}{*}{$N(\%)$} & \multicolumn{2}{|c|}{ Month 4} & \multicolumn{2}{|c|}{ Month 12} & \multicolumn{2}{|c|}{ Month 24} \\
\hline & & $n(\%)$ & $p$-value & $n(\%)$ & $p$-value & $n(\%)$ & $p$-value \\
\hline \multicolumn{8}{|l|}{ Age, years } \\
\hline $10-14$ & $41(18.6)$ & $29(70.7)$ & 0.106 & $26(63.4)$ & $0.001^{*}$ & $24(58.5)$ & $<0.001^{\star}$ \\
\hline $15-19$ & $179(81.4)$ & $102(57.0)$ & & $62(34.6)$ & & $31(17.3)$ & \\
\hline \multicolumn{8}{|l|}{ Sex } \\
\hline Male & $38(17.3)$ & $31(81.6)$ & $0.002^{*}$ & $21(55.3)$ & $0.035^{\star}$ & $18(47.4)$ & $<0.001^{*}$ \\
\hline Female & $182(82.7)$ & $100(54.9)$ & & $67(36.8)$ & & $37(20.3)$ & \\
\hline \multicolumn{8}{|l|}{ Source of income } \\
\hline Employed & $15(9.4)$ & $10(66.7)$ & 0.141 & $5(33.3)$ & 0.365 & $2(13.3)$ & $0.003^{*}$ \\
\hline Family and friends & $129(80.6)$ & $73(56.6)$ & & $51(39.5)$ & & $25(19.4)$ & \\
\hline Grant & $16(10.0)$ & $13(81.3)$ & & $9(5.6)$ & & $9(5.6)$ & \\
\hline \multicolumn{8}{|l|}{ Type of dwelling } \\
\hline Informal & $69(36.1)$ & $42(60.9)$ & 0.288 & $27(39.1)$ & 0.454 & $14(20.3)$ & 0.589 \\
\hline Formal house & $116(60.7)$ & $64(55.2)$ & & $43(37.1)$ & & $27(23.3)$ & \\
\hline Hostel & $4(2.1)$ & $1(25.0)$ & & 0 & & 0 & \\
\hline Other & $2(1.1)$ & $2(100)$ & & $1(50)$ & & 0 & \\
\hline \multicolumn{8}{|c|}{ Disclosure to significant other } \\
\hline Yes & $182(87.1)$ & $108(59.3)$ & 0.709 & $76(41.8)$ & 0.117 & $49(26.9)$ & 0.076 \\
\hline No & $27(12.9)$ & $15(55.6)$ & & $7(25.9)$ & & $3(11.1)$ & \\
\hline \multicolumn{8}{|c|}{ Reported alcohol and/or drug use } \\
\hline Yes & $27(15)$ & $16(59.3)$ & 0.721 & $10(37.0)$ & 0.913 & $4(14.8)$ & 0.510 \\
\hline No & $153(85)$ & $85(55.6)$ & & $55(35.9)$ & & $31(20.3)$ & \\
\hline \multicolumn{8}{|l|}{ CD 4 count, cells $/ \mu \mathrm{L}$} \\
\hline$<200$ & $42(19.3)$ & $24(57.1)$ & 0.703 & $18(42.9)$ & 0.816 & $14(33.3)$ & 0.262 \\
\hline $200-349$ & $109(50.0)$ & $64(58.7)$ & & $45(41.3)$ & & $28(25.7)$ & \\
\hline$\geq 350$ & $67(30.7)$ & $43(64.2)$ & & $25(37.3)$ & & $13(19.4)$ & \\
\hline \multicolumn{8}{|l|}{ WHO stage } \\
\hline I & $99(46.5)$ & $51(51.5)$ & 0.081 & $35(35.4)$ & 0.441 & $15(15.2)$ & $0.020^{*}$ \\
\hline II & $48(22.5)$ & $30(62.5)$ & & $22(45.8)$ & & $15(31.3)$ & \\
\hline III & $51(23.9)$ & $37(72.5)$ & & $20(39.2)$ & & $17(33.3)$ & \\
\hline IV & $15(7.0)$ & $10(66.7)$ & & $8(53.3)$ & & $6(40.0)$ & \\
\hline \multicolumn{8}{|l|}{ TB treatment } \\
\hline Yes & $31(14.1)$ & $23(74.2)$ & 0.073 & $15(48.4)$ & 0.304 & $11(35.5)$ & 0.146 \\
\hline No & $189(85.9)$ & $108(57.1)$ & & $73(38.6)$ & & $44(23.3)$ & \\
\hline \multicolumn{8}{|l|}{ Pregnant } \\
\hline Yes & $84(38.2)$ & $39(46.4)$ & $0.002^{*}$ & $25(29.8)$ & $0.015^{*}$ & $7(8.3)$ & $<0.001^{\star}$ \\
\hline No & $136(61.8)$ & $92(67.6)$ & & $63(46.3)$ & & $48(35.3)$ & \\
\hline \multicolumn{8}{|l|}{ IPT } \\
\hline Yes & $15(6.8)$ & $8(53.3)$ & 0.492 & $5(33.3)$ & 0.826 & $2(13.3)$ & 0.184 \\
\hline No & $165(73.2)$ & $96(58.2)$ & & $66(40.0)$ & & $39(23.6)$ & \\
\hline \multicolumn{8}{|l|}{ Co-trimoxazole } \\
\hline Yes & $84(38.2)$ & $55(65.5)$ & 0.203 & $38(45.2)$ & 0.437 & $30(35.7)$ & $0.010^{*}$ \\
\hline No & $51(61.8)$ & $26(50.0)$ & & $20(38.5)$ & & $12(23.1)$ & \\
\hline \multicolumn{8}{|l|}{ ART regimen } \\
\hline TFE & $157(71.4)$ & $88(56.1)$ & 0.296 & $54(34.4)$ & $0.019^{*}$ & $25(15.9)$ & $<0.001^{\star}$ \\
\hline $\mathrm{T} 3 \mathrm{E}$ & $27(12.3)$ & $16(59.3)$ & & $11(40.7)$ & & $7(25.9)$ & \\
\hline A3E & $34(15.5)$ & $25(73.5)$ & & $21(61.8)$ & & $22(64.7)$ & \\
\hline $\mathrm{Z3E}$ & $1(0.4)$ & $1(100)$ & & $1(100)$ & & 0 & \\
\hline T3L/rit & $1(0.4)$ & $1(100)$ & & $1(100)$ & & $1(100)$ & \\
\hline
\end{tabular}

as $59.5 \%$ v. $86.8 \%, 40 \%$ v. $79.3 \%$ and $25 \%$ v. $68.8 \%$, respectively. Therefore, VLS was significantly associated with retention in care.
However, VLS fell short of the targeted 90\% throughout the study observation period. 
Table 2 shows that younger adolescents (10 - 14 years) had a higher risk of VLS at 12 and 24 months post initiation on ART than older adolescents (15 - 19 years). At 12 months post initiation on ART, younger adolescents had an $83 \%$ higher risk of VLS (risk ratio (RR) 1.83 (95\% CI 1.35 - 2.49)) than older adolescents; and $>3$ times higher risk (RR 3.38 (95\% CI $2.24-5.10)$ ) at 24 months.

Male adolescents had a higher risk of VLS at 4 months (RR 1.49 (95\% CI 1.22 - 1.81)), 12 months (RR 1.5 (95\% CI 1.07 - 2.12)) and 24 months (RR $2.33(1.50-3.62)$ ) post initiation than female adolescents.

Adolescents who were pregnant had a significantly lower risk of VLS than all other adolescents (RR 0.69 (95\% CI $0.53-0.89)$ ) at 6 months; RR 0.64 (95\% CI 0.44 - 0.94) at 12 months; and RR 0.24 (at all the observed time points).

There was no statistically significant difference in achieving VLS at 4 and 12 months post initiation among adolescents, based on WHO staging at initiation. However, there was a statistically significant difference in VLS at 24 months post initiation on treatment for adolescents who were classified as WHO stage I at initiation compared with those classified as WHO stage II, III or IV $(p=0.020)$. At 24 months post-ART initiation, those who were at WHO stage II at initiation had a 52\% higher risk of VLS (RR 1.52 (95\% CI 1.09 1.74)), those who were WHO stage III - 55\% (RR 1.55 (95\% CI 1.17 - 1.75)) and those who were WHO stage IV - 62\% (RR 1.62 (95\% CI 1.18 - 1.83)) compared with those who were initiated on ART at WHO stage I.

\section{Discussion}

In 2014, the WHO set a global target of 90-90-90, which required that by $2020,90 \%$ of all people living with HIV are diagnosed, $90 \%$ of those diagnosed are initiated on ART, and $90 \%$ of those on ART achieve viral suppression. ${ }^{[1]}$ Our study cohort fell short of this target throughout the observation period, with rates of 59.5\%, $40.0 \%$ and $25.0 \%$ at month 4,12 and 24 post initiation, respectively. Even among those retained in care, VLS rates were unsatisfactory, i.e. 86.8\%, 79.3\% and $68.8 \%$ at month 4,12 and 24 post initiation, respectively. These results indicate poor ART adherence of adolescents, in addition to the significant loss to follow-up. Our results follow the trends reported elsewhere in SSA and other regions, ${ }^{[8]}$ giving further credence to the global importance of addressing unsatisfactory treatment outcomes for adolescents receiving ART.

Older adolescents (15 - 19 years) were observed to have lower rates of VLS than younger adolescents. This is an important finding, and emphasises that adolescents experience significant adherence problems when transitioning into adult HIV programmes. Although very few facilities in the Western Cape provide separate paediatric and adult treatment programmes, adolescent patients are treated differently once their HIV status has been disclosed to them, and they are expected to self-manage their HIV condition (as an adult). There are very few youth clubs for adolescents and young people in the Western Cape Metropole. Where these are offered, limited psychosocial support is effectively delivered as part of the service; and these become de facto quick medication pick-up services only.

A surprising finding from our study was that male adolescents had higher rates of VLS than females. It should be noted that only $46 \%$ of female adolescents were initiated on ART while pregnant as part of the prevention of mother-to-child transmission (PMTCT) programme. Their rates of VLS were consistently lower compared with all other adolescents; at 24 months, only $8 \%$ had VLS. This finding concurs with findings in other studies, where adherence, retention in care and VLS decrease significantly after 12 months, which corresponds to the completion of the immunisation schedule for an infant. The PMTCT programme booking provides opportunities to support the pregnant adolescent to achieve favourable outcomes for her and her baby. These opportunities include having a longer time period to educate the patient on HIV and ART, as well as identifying and assisting with any barriers to poor adherence, including disclosure or socioeconomic challenges. Early antenatal care booking becomes increasingly important in the context of the Western Cape ART guidelines that encourage same-day ART initiation for pregnant HIV-infected women. ${ }^{[12]}$

Adolescents in our study who were initiated at higher WHO stages (II, III and IV) at baseline had higher rates of VLS, although this did not reach statistical significance, except at month 24 . Therefore, just fewer than half of the cohort did not feel particularly ill when they were enrolled on ART, and were therefore not motivated by ill-health to remain fully adherent and engaged in care. With the introduction of universal coverage for HIV-infected individuals, irrespective of

Table 2. Rate of viral load suppression among adolescents initiated on ART in Metro District Health Services facilities in the Western Cape, South Africa, $2013(N=220)$

\begin{tabular}{|c|c|c|c|}
\hline & Month 4 & Month 12 & Month 24 \\
\hline & RR $(95 \%$ CI $)$ & RR $(95 \% \mathrm{CI})$ & RR $(95 \%$ CI $)$ \\
\hline \multicolumn{4}{|l|}{ Age, years } \\
\hline $10-14$ & $1.24(0.98-1.57)$ & $1.83(1.35-2.49)$ & $3.38(2.24-5.10)$ \\
\hline $15-19$ & 1.00 & 1.00 & 1.00 \\
\hline \multicolumn{4}{|l|}{ Sex } \\
\hline Male & $1.49(1.22-1.81)$ & $1.50(1.07-2.12)$ & $2.33(1.50-3.62)$ \\
\hline Female & 1.00 & 1.00 & 1.00 \\
\hline \multicolumn{4}{|c|}{ WHO stage } \\
\hline I & 1.00 & 1.00 & 1.00 \\
\hline II & $1.18(0.90-1.38)$ & $1.23(0.84-1.49)$ & $1.52(1.09-1.74)$ \\
\hline III & $1.29(1.08-1.45)$ & $1.10(0.61-1.42)$ & $1.55(1.17-1.75)$ \\
\hline IV & $1.23(0.84-1.49)$ & $1.34(0.86-1.62)$ & $1.62(1.18-1.83)$ \\
\hline \multicolumn{4}{|l|}{ Pregnant } \\
\hline Yes & $0.69(0.53-0.89)$ & $0.64(0.44-0.94)$ & $0.24(0.11-0.50)$ \\
\hline No & 1.00 & 1.00 & 1.00 \\
\hline
\end{tabular}


WHO staging or CD4 count, ${ }^{[12]}$ relatively healthier people will be initiating ART, resulting in the potential risk of healthier people not being motivated to remain in care, as reported in another study. ${ }^{[13]}$ Therefore, the ART programme needs to scale up efforts to motivate patients to remain in care and continue taking their medication to achieve VLS.

\section{Study limitations}

There are some important clinical parameters, such as comorbidities, duration of infection (diagnosis) and mode of acquisition of HIV that are covariates for the outcome measure of VLS, which were not obtained from medical records, as these were poorly and inconsistently captured.

\section{Conclusions}

All adolescents in our study had viral loads taken and recorded while they remained in care - this speaks to a measure of quality of care being provided in public health facilities in the Western Cape Metropole. Our study highlights the low rates of VLS among adolescents, which deserve urgent attention to improve adherence and retention in care for adolescents. Such interventions should address the unique needs of older adolescents and adolescents who initiate ART while pregnant. Behavioural interventions to improve adherence should include positive messaging about health and wellbeing, rather than the prevention of illness and death.

\section{Declaration. None.}

Acknowledgements. The authors thank the staff at the participating health facilities.

Author contributions. BEvW and EK conceptualised the study; EK, with support from BEvW and FCM, analysed the data; BEvW wrote the manuscript; and BEvW, FCM and EK reviewed the manuscript.
Funding. This study was supported by the South African Medical Research Council (SA MRC) under a Self-Initiated Research Grant. The views and opinions expressed are those of the authors and do not necessarily represent the official views of the SA MRC.

Conflicts of interest. None.

1. United Nations Children's Fund (UNICEF). Turning the tide against AIDS will require more concentrated focus on adolescents and young people. 2018. https://data.unicef.org/topic/adolescents/ hiv-aids (accessed 28 August 2019).

2. United Nations Children's Fund (UNICEF). Children, adolescents and AIDS. 2014. http:// childrenandaids.org/files/Stats_Update_11-27.pdf (accessed 18 August 2018).

3. World Health Organization (WHO). Health for the world's adolescents: A second chance in the second World Health Organization (WHO). Health for the world's adolescents: A second chance in the second
decade. 2014. http://apps.who.int/adolescent/second-decade/files/1612_MNCAH_HWA_Executive_ decade. 2014. http://apps. who.int/ado.
Summary.pdf (accessed 16 July 2017).

4. Msemburi W, Pillay-van Wyk V, Dorrington RE, et al. Second National Burden of Disease Study for South Africa: Cause-of-Death Profile for South Africa, 1997 -2012. Cape Town: South African Medical Research Council, 2016

5. Simbayi LC, Zuma K, Zungu N, et al. South African National HIV Prevalence, Incidence, Behaviour and Communication Survey, 2017. Cape Town: Human Sciences Research Council, 2018.

6. Kim SH, Gerver SM, Fidler S, et al. Adherence to antiretroviral therapy in adolescents living with HIV: Systematic review and meta-analysis. AIDS 2014;28(13):1945-1956. https://doi.org/10.1097/ qad. 0000000000000316

7. Ferrand RA, Briggs D, Ferguson J, et al. Viral suppression in adolescents on antiretroviral treatment: Review of the literature and critical appraisal of methodological challenges. Trop Med Int Health 2016;21(3):325-333. https://doi.org/10.1111/tmi.12656

8. Nglazi MD, Kranzer K, Holele P, et al. Treatment outcomes in HIV-infected adolescents attending a Nglazi MD, Kranzer K, Holele P, et al. Treatment outcomes in HIV-infected adolescents attending a
community-based antiretroviral therapy clinic in South Africa. BMC Infect Dis 2012;12:21. https:// community-based antiretroviral

9. Nachega JB, Hislop M, Nguyen H, et al. Antiretroviral therapy adherence, virologic and immunologic outcomes in adolescents compared with adults in southern Africa. J AIDS 2009;51(1):65-71. https:/ doi.org/10.1097/QAI.0b013e318199072

10. Zanoni BC, Archary M, Buchan S, et al. Systematic review and meta-analysis of the adolescent HIV continuum of care in South Africa: The cresting wave. BMJ Glob Health 2016;1(3):e000004. https:// doi.org/10.1136/bmjgh-2015-000004

1. World Health Organization. 90-90-90. An ambitious treatment target to help end the AIDS epidemic. https://www.unaids.org/sites/default/files/media_asset/90-90-90_en.pdf (accessed 18 October 2018).

12. Western Cape Government Health. The Western Cape Consolidated Guidelines for HIV Treatment: Prevention of Mother-to-Child Transmission of HIV (PMTCT), Children, Adolescents and Adults. Cape Town: WCGH, 2016.

13. Ngarina M, Popenoe R, Kilewo $C$, et al. Reasons for poor adherence to antiretroviral therapy postnatally in HIV-1 infected women treated for their own health: Experiences from the Mitra Plus study in Tanzania. BMC Publ Health 2013;13(1):450. https://doi.org/10.1186/1471-2458-13-450 